GaN nano-pyramid arrays as an efficient photoelectrode for solar water splitting

This content has been downloaded from IOPscience. Please scroll down to see the full text.

2016 Nanotechnology 27455401

(http://iopscience.iop.org/0957-4484/27/45/455401)

View the table of contents for this issue, or go to the journal homepage for more

Download details:

IP Address: 143.167.30.63

This content was downloaded on 30/11/2016 at 15:26

Please note that terms and conditions apply.

You may also be interested in:

Enhanced water splitting with silver decorated GaN photoelectrode

Y Hou, Z A Syed, R Smith et al.

InP nanopore arrays for photoelectrochemical hydrogen generation

Qiang Li, Maojun Zheng, Bin Zhang et al.

Controlled synthesis of GaN-based nanowires for photoelectrochemical water splitting applications Mohamed Ebaid, Jin-Ho Kang and Sang-Wan Ryu

High efficiency photoelectrochemical water splitting and hydrogen generation using GaN nanowire photoelectrode

B AlOtaibi, M Harati, S Fan et al.

Photoelectrochemical properties of InN nanowire photoelectrodes for solar water splitting

J Kamimura, P Bogdanoff, M Ramsteiner et al.

Plasmon-enhanced nanoporous BiVO4 photoanodes for efficient photoelectrochemical water oxidation Jiayong Gan, Bharath Bangalore Rajeeva, Zilong Wu et al.

Facile approaching hierarchical CdS films as electrode toward photoelectrochemical water splitting Xin Guo, Junjun Zhu, Huaixin Wei et al.

Constructing n-ZnO@Au heterogeneous nanorod arrays on p-Si substrate as efficient photocathode for water splitting

Zhijia Bao, Xiaoyong Xu, Gang Zhou et al. 


\title{
GaN nano-pyramid arrays as an efficient photoelectrode for solar water splitting
}

\author{
Y Hou, X Yu, Z Ahmed Syed, S Shen, J Bai and T Wang \\ Department of Electrical and Electronic Engineering, University of Sheffield, Mappin Street, Sheffield S1 \\ 3JD, UK \\ E-mail: t.wang@sheffield.ac.uk
}

Received 2 August 2016, revised 4 September 2016

Accepted for publication 12 September 2016

Published 11 October 2016

\begin{abstract}
A prototype photoelectrode has been fabricated using a GaN nano-pyramid array structure grown on a cost-effective $\mathrm{Si}$ (111) substrate, demonstrating a significant improvement in performance of solar-powered water splitting compared with any planar GaN photoelectrode. Such a nanopyramid structure leads to enhanced optical absorption as a result of a multi-scattering process which can effectively produce a reduction in reflectance. A simulation based on a finitedifference time-domain approach indicates that the nano-pyramid architecture enables incident light to be concentrated within the nano-pyramids as a result of micro-cavity effects, further enhancing optical absorption. Furthermore, the shape of the nano-pyramid further facilitates the photo-generated carrier transportation by enhancing a hole-transfer efficiency. All these features as a result of the nano-pyramid configuration lead to a large photocurrent of $1 \mathrm{~mA} \mathrm{~cm}^{-2}$ under an illumination density of $200 \mathrm{~mW} \mathrm{~cm}^{-2}$, with a peak incident photon-to-current conversion efficiency of $46.5 \%$ at $\sim 365 \mathrm{~nm}$, around the band edge emission wavelength of GaN. The results presented are expected to pave the way for the fabrication of $\mathrm{GaN}$ based photoelectrodes with a high energy conversion efficiency of solar powered water splitting.
\end{abstract}

Keywords: photoelectrode, water splitting, hydrogen generation, GaN

(Some figures may appear in colour only in the online journal)

\section{Introduction}

Solar-powered water splitting would be the most promising approach to converting solar energy into renewable and storable energy. III-nitride semiconductors ( $\mathrm{GaN}$ and its alloys such as $\mathrm{InGaN}$ ) exhibit a wide range of bandgap from $3.4 \mathrm{eV}$ of $\mathrm{GaN}$ to $0.69 \mathrm{eV}$ of $\mathrm{InN}[1,2]$, covering the whole visible spectral range and thus matching the solar spectrum perfectly. Furthermore, it has been predicted that the conduction band minimum and the valence band maximum of $\mathrm{In}_{x} \mathrm{Ga}_{1-x} \mathrm{~N}$ with a wide range of indium content $(x<50 \%)$ can straddle over the redox potentials of $\left(\mathrm{H}^{+} / \mathrm{H}_{2}\right)$ and $\left(\mathrm{O}_{2} / \mathrm{H}_{2} \mathrm{O}\right)$ for overall water splitting [3]. All these unique properties along with their excellent chemical stability in acidic or alkaline solutions which is crucial for water splitting make III-nitrides be ideal candidates for solar-powered hydrogen generation $[4,5]$. The last two decades major efforts have been devoted to the development of III-nitride based emitters for solid state lighting represented by InGaN based blue emitters, leading to the award of the Nobel Prize in 2014. It is good timing to attempt to extend the utilisation of $\mathrm{GaN}$ and its alloys to the field of solar-powered hydrogen generation in a cost-effective manner, given a number of the major advantages mentioned above.

Current researches on $\mathrm{GaN}$ based photoelectrodes are in infancy, and thus the solar-hydrogen conversion efficiency is not impressive so far. In order to achieve a step-change in efficiency of solar-powered hydrogen generation, a number of major challenges will have to be met, such as enhanced light absorption, rapid migration of the charged carriers photongenerated to the semiconductor/electrolyte junction in order to participate in the half-reactions before their recombination. So far, a number of ideas using nanostructures have been proposed, such as nanowires or nanorods, nanoporous 

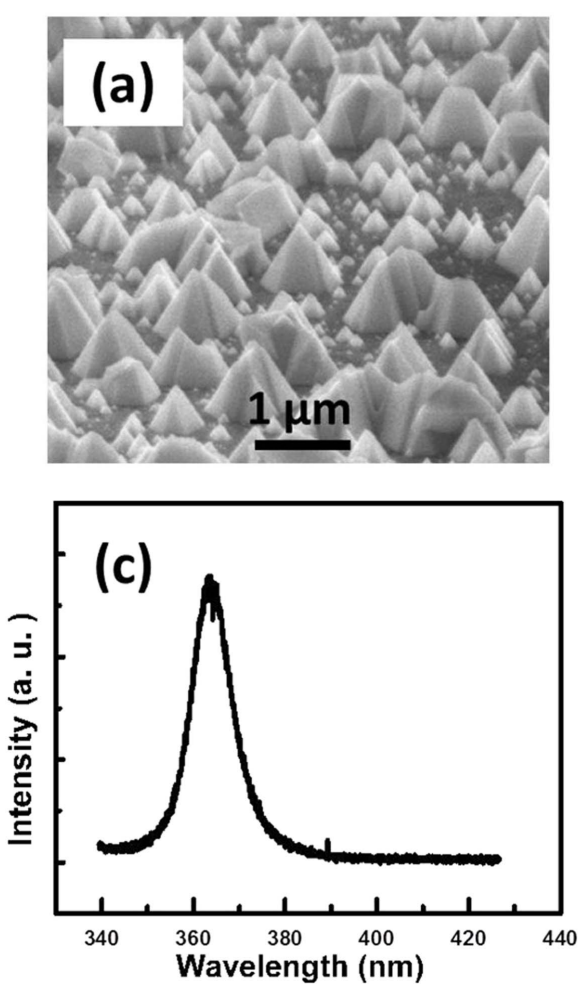

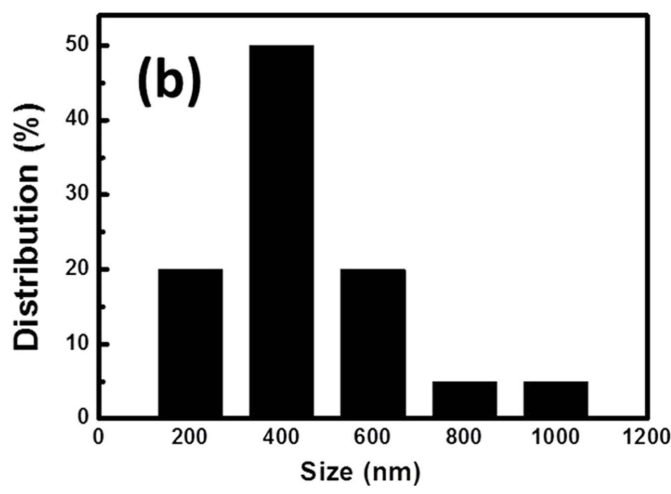

(d)

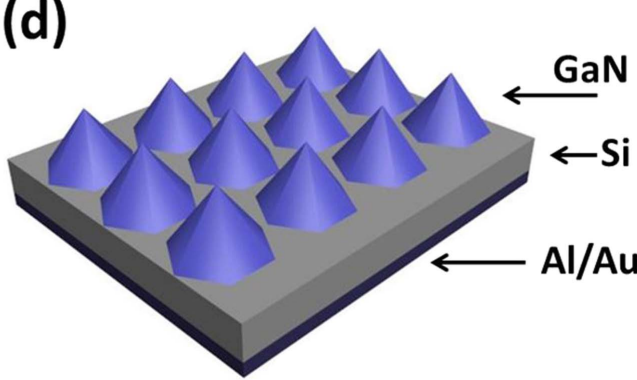

Figure 1. (a) SEM of our GaN nano-pyramids grown on Si (111); (b) typical base size distribution of our GaN nano-pyramids; (c) roomtemperature PL spectrum of our GaN nano-pyramids; and (d) schematics of our photoelectrode structure using the GaN nano-pyramids.

structures [6-9]. As a consequence of the increased surfaceto-volume ratio resulting from nanostructures, optical absorption can be significantly enhanced. The migration of photon-generated carriers is mainly determined by the diffusion length of minority carriers and the distance they must travel. When diffusion length is short or a travelling distance is long, minority carriers will recombine before they reach the junction. A nanostructure configuration would lead to a reduction in travelling distance for the photon-generated carriers to the semiconductor/electrolyte junction, thus potentially enhancing energy conversion efficiency.

In the present work, we report a prototype photoelectrode fabricated using a GaN nano-pyramid array structure, grown on cost-effective Si (111) substrates by metal organic chemical vapour deposition (MOCVD). Such a nano-pyramid structure is expected to enhance optical absorption as a result of a multi-scattering process which can effectively produce a reduction in reflectance [10]. Furthermore, a simulation based on a finite-difference time-domain (FDTD) approach demonstrates that this architecture enables incident light to be concentrated inside the nano-pyramids, working as nanoconcentrators which can further enhance light harvesting. The unique shape of the nano-pyramids leads to an enhanced holetransfer efficiency, further enhancing the device performance. As a result, our device demonstrates a large photocurrent of $\sim 1 \mathrm{~mA} \mathrm{~cm}^{-2}$ under an illumination of $200 \mathrm{~mW} \mathrm{~cm}^{-2}$, with a peak incident photon-to-current conversion efficiency of $46.5 \%$ at $365 \mathrm{~nm}$, around the band edge emission wavelength of $\mathrm{GaN}$.

\section{Fabrication}

The GaN nano-pyramid structures were grown on n-type $\mathrm{Si}$ (111) substrates by a low pressure MOCVD system. The substrates were initially subject to a thermal cleaning process at $1145{ }^{\circ} \mathrm{C}$ in ambient $\mathrm{H}_{2}$ in order to remove any oxides, and a thin AlN layer was then grown as a nucleation layer also at $1145^{\circ} \mathrm{C}$. Afterward, the growth temperature was ramped down to $800^{\circ} \mathrm{C}$ for the growth of a nano-pyramid array structure. Figure 1(a) presents a typical scanning electron microscopy image of our nano-pyramid $\mathrm{GaN}$ grown on $\mathrm{Si}$ (111). The density of the nano-pyramids is $\sim 5 \times 10^{8} \mathrm{~cm}^{-2}$, and the size (measured from the base of individual pyramid) ranges from $200 \mathrm{~nm}$ up to $1 \mu \mathrm{m}$. Figure 1(b) shows a typical base size distribution of our nano-pyramids, exhibiting that it is dominated by the nano-pyramids with a base size of $400 \mathrm{~nm}$. X-ray diffraction measurements performed in a $2 \theta / \Omega$ mode confirm that our nano-pyramids typically orient along the (0001) direction. Optical properties were characterised by standard photoluminescence (PL) measurements performed at room temperature using a $325 \mathrm{~nm} \mathrm{He}-\mathrm{Cd}$ laser with a $0.75 \mathrm{~m}$ monochromator. A strong band emission has been observed at $363 \mathrm{~nm}$ as shown in figure 1(c). In order to fabricate a photoelectrode, an $\mathrm{Al} / \mathrm{Au}(100 / 100 \mathrm{~nm})$ alloy was deposited onto the back of the Si substrate, followed by a rapid thermal annealing process at $600{ }^{\circ} \mathrm{C}$ in $\mathrm{N}_{2}$ for $1 \mathrm{~min}$ to form an ohmic contact. Our device structure is schematically illustrated in figure 1(d). For solar-powered water splitting measurements, a copper wire was bonded on the ohmic contact with silver 

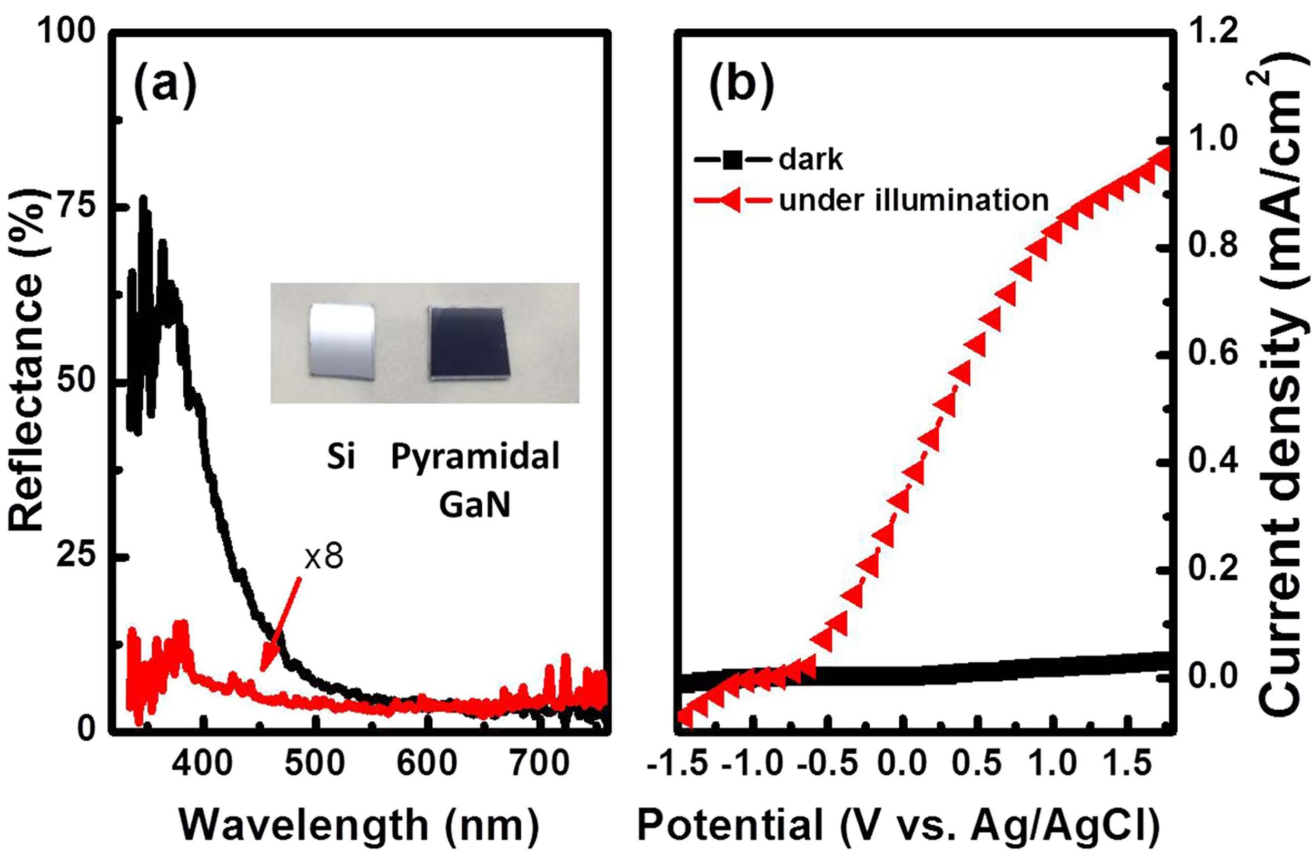

Figure 2. (a) Reflectance of GaN nano-pyramids and Si substrate. The inset shows the photographs of a piece of the Si substrate and a piece of the GaN nano-pyramid structure grown on $\mathrm{Si}$. (b) Photocurrent density of our photoelectrode fabricated from the GaN nano-pyramids, measured under an illumination of $200 \mathrm{~mW} \mathrm{~cm}^{-2}$.

epoxy. The whole contact area was covered by an insulating epoxy for protection as usual.

\section{Results and discussion}

Photocurrent measurements have been performed using a source-metre (Keithley 2401), which connects the working photoelectrode and a $\mathrm{Pt}$ counter electrode placed inside an electrolyte solution $(\mathrm{HCl}, 1 \mathrm{M}) . \mathrm{Ag} / \mathrm{AgCl}$ has been applied as a reference electrode. The illumination source used in our experiment is a standard LOT-Oriel solar simulator with a tuneable $300 \mathrm{~W}$ ozone free Xe arc lamp in combination with an AM 1.5 filter. In order to have a straightforward visual comparison, a piece of $\mathrm{Si}$ substrate without GaN nano-pyramids is provided as a reference. The reflectance curves of the two devices are shown in figure 2(a). Obviously, the reflectance in the UV region is massively reduced due to scattering and absorption by the GaN nano-pyramids. Inset of the figure 2(a) shows the photo-graphs of the two devices for comparison. Due to the reduced reflectance as a result of the nano-pyramid configuration, our sample exhibits complete dark colour compared to the shining Si substrate. The photocurrent density has been measured under a bias ranging from -1.5 to $1.8 \mathrm{~V}$ versus the reference electrode (versus $\mathrm{Ag}$ / $\mathrm{AgCl}$ ), with a step of $0.1 \mathrm{~V}$ under an illumination density of $200 \mathrm{~mW} \mathrm{~cm}^{-2}$. The photocurrent starts to appear under a bias at $-0.6 \mathrm{~V}$, which is close to the value previously reported on other $\mathrm{GaN}$ based photoelectrodes [6]. When the bias is above $-0.6 \mathrm{~V}$, the photocurrent density increases quickly with further increasing bias, with a saturation value of $\sim 1 \mathrm{~mA} \mathrm{~cm}^{-2}$, which is about five times higher than that of a planar $\mathrm{GaN}$ photoelectrode on sapphire under a same condition [11].

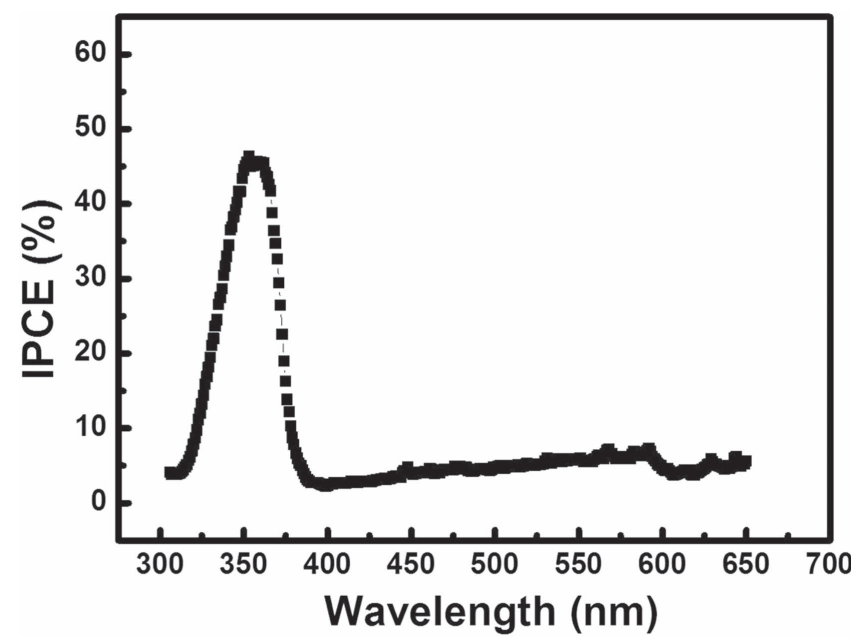

Figure 3. IPCE of photoelectrode fabricated from the GaN nanopyramids as a function of wavelength at $0 \mathrm{~V}$.

An applied bias photon-to-current conversion efficiency (ABPE) can be calculated using the equation (1) below

$$
\eta=\frac{j_{\mathrm{p}}\left(\mathrm{mA} \mathrm{cm}^{-2}\right)\left(E_{\mathrm{rev}}^{0}-V_{\mathrm{ext}}\right)}{I\left(\mathrm{~mW} \mathrm{~cm}^{-2}\right)} \times 100 \%,
$$

where $J_{\mathrm{p}}, V_{\mathrm{ext}}, I$ and $E_{\mathrm{rev}}^{0}$ are the photocurrent density, the external bias, the illumination density and the redox potential of the electrolyte used (for $1 \mathrm{M} \mathrm{HCl}, E_{\mathrm{rev}}^{0}=1.4 \mathrm{~V}$ ), respectively. For simplicity, a case under an external bias of $0.6 \mathrm{~V}$ has been considered, where the corresponding photocurrent density is $0.68 \mathrm{~mA} \mathrm{~cm}^{-2}$. Consequently, the ABPE is estimated to be $0.27 \%$. Such an ABPE is not low, given that only the ultraviolet part with a wavelength shorter than $363 \mathrm{~nm}$ (or photon energy $>3.4 \mathrm{eV}$, i.e., the $\mathrm{GaN}$ bandgap) can be 

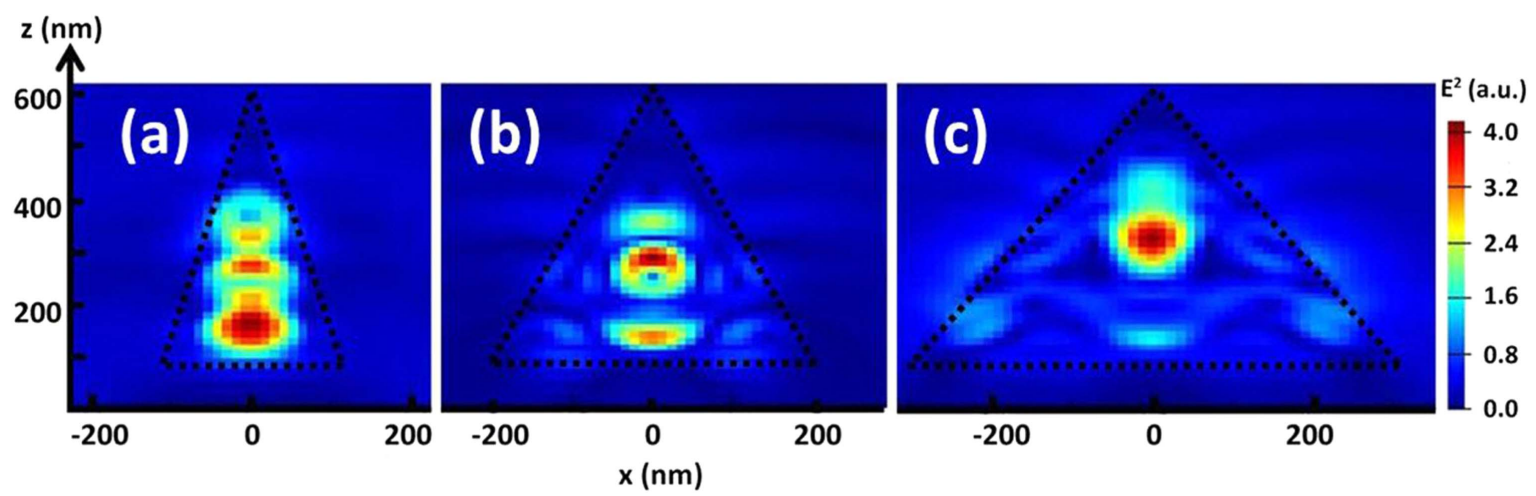

Figure 4. Distribution of the incident light intensity within the nano-pyramids with a size ranging from $200 \mathrm{~nm}$ (a), $400 \mathrm{~nm}$ (b) and $600 \mathrm{~nm}(\mathrm{c})$, respectively.

effectively absorbed. This accounts for less than $4 \%$ of the whole solar spectrum.

The incident photon-to-current efficiency (IPCE) characterises the capability of converting electrical power from the absorbed light at each corresponding wavelength. It can be mathematically described by equation (2) below

$$
\mathrm{IPCE}=\frac{1240 \times J\left(\mathrm{~mA} \mathrm{~cm}^{-2}\right)}{\lambda(\mathrm{nm}) \times I\left(\mathrm{~mW} \mathrm{~cm}^{-2}\right)} \times 100 \%,
$$

where $J, \lambda$ and $I$ are the photocurrent density, the incident light wavelength and the density of incident light.

The IPCE measurements were performed at $0 \mathrm{~V}$ using a $75 \mathrm{~W}$ Xe lamp as an illumination source with a $0.22 \mathrm{~m}$ monochromator, whilst the photocurrent was recorded by a source-metre (Keithley 2401). Figure 3 shows a typical IPCE of our nano-pyramid $\mathrm{GaN}$ photoelectrode as a function of wavelength, demonstrating a peak IPCE of $46.5 \%$ at $365 \mathrm{~nm}$, around the band edge emission wavelength of $\mathrm{GaN}$. There is no any response observed from $\mathrm{Si}$, indicating the photocurrent is contributed by the GaN nano-pyramids. The IPCE of $46.5 \%$ is much higher than that of any photoelectrode fabricated from any standard planar GaN on sapphire $(\sim 15 \%)$ $[10,12]$, indicating excellent performance of our nano-pyramid GaN photoelectrode. Compared with the InGaN/GaN nanowires photolelectrodes reported by other groups [13, 14], where the density of the nanowires is much higher high than that of our nano-pryamids, the IPCE of our nano-pyramid array photoelectrode is lower but not far away. This means that we have a plenty of scopes to further improve the performance of our devices.

In order to understand the mechanism of the enhanced performance of our nano-pyramid GaN photoelectrode, a simulation has been performed. The distribution of the incident light intensity in our device has been studied by a standard FDTD simulation, where the refractive index of GaN and the incident light wavelength are set to be 2.5 and $300-400 \mathrm{~nm}$, respectively. The electric field profiles of the incident light within the nano-pyramids with a size of 200 , 400 and $600 \mathrm{~nm}$ have been calculated, respectively, as shown in figure 4. In each case, the simulation shows clear microcavity effects, meaning significantly enhanced incident light distributed within the nano-pyramid as a result of its nanopyramids geometry. Consequently, it leads to a further increase in optical absorption in addition to an increased surface-to-volume ratio. In addition, the surface of the nanopyramids also reduces optical reflectance, further enhancing sunlight harvesting.

In addition to the enhanced light absorption due to the aforementioned reasons, an inhomogeneous electric field (nanojunction) is expected to be formed at the $\mathrm{GaN}$ nanopyramid/electrolyte interface [15]. Unlike the planar device, the strong static electric field distributes along both axial and radial directions of the nano-pyramids. As a result, the minority carriers (holes) will be effectively separated from photogenerated electron-hole pairs and driven into electrolyte in all directions from the nano-pyramids with a large speed $\left(v=\mu_{\mathrm{h}} E\right)$, generating a high hole transfer efficiency.

Transient photocurrent measurements have been performed under a periodic illumination of $300 \mathrm{~mW} \mathrm{~cm}^{-2}$ in order to further study the carrier transfer efficiency of our device. As shown in figure 5(a), the photocurrent appears a repeatable 'spike and overshoot' when the illuminating source used is 'on/off'. This phenomenon has been often observed on both oxide and nitride semiconductor based photoelectrodes $[1,16]$. However, it has rarely been discussed on GaN based photoelectrodes. This is attributed to a complicated carrier transportation process, which is mainly related to a hole-transfer efficiency from a photoelectrode to electrolyte [17]. With an empirical equation, the hole-transfer efficiency labelled as $\eta_{j}$ can be described below: [17]

$$
\eta_{j}=\frac{j_{\mathrm{s}}}{j_{t=0}} \times 100 \%
$$

where $j_{\mathrm{s}}$ and $j_{t}=0$ are the steady state photocurrent and the initial photocurrent when the light is switched on, respectively. Based on the data provided in figure 5(a), the holetransfer efficiency is estimated to be $\sim 85 \%$. High holetransfer efficiency indicates a short transit time $(t)$ of the photo-generated carriers. In the case of $t<\tau$ (excess carrier lifetime), which means the carrier can circulate more than once in the circuits, likely generating a current gain [18].

The photocurrent stability of our photoelectrode has been investigated in a continuous working mode, measured under an illumination intensity of $300 \mathrm{~W} \mathrm{~cm}^{-2}$ under $0.6 \mathrm{~V}$ in $1 \mathrm{M}$ $\mathrm{HCl}$. Figure 5(b) exhibits the photocurrent and dark current densities as a function of time. The photocurrent density starts 

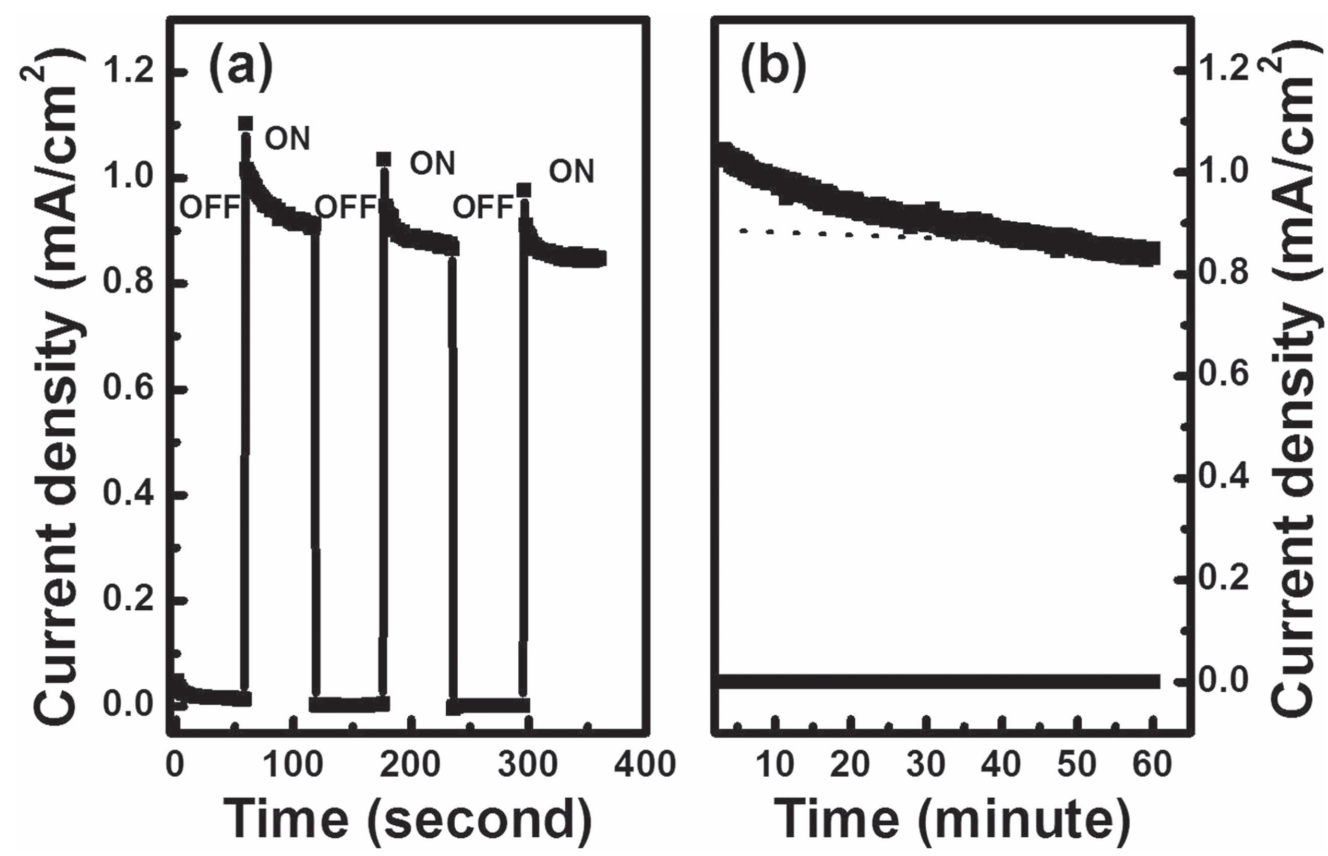

Figure 5. (a) Transient photocurrent characterisation as a function of time under a $300 \mathrm{~mW} \mathrm{~cm}{ }^{-2}$ illumination density in a pulsed mode; and (b) photocurrent as a function of time under an illumination density of $300 \mathrm{~mW} \mathrm{~cm}^{-2}$ in a continuous working mode.

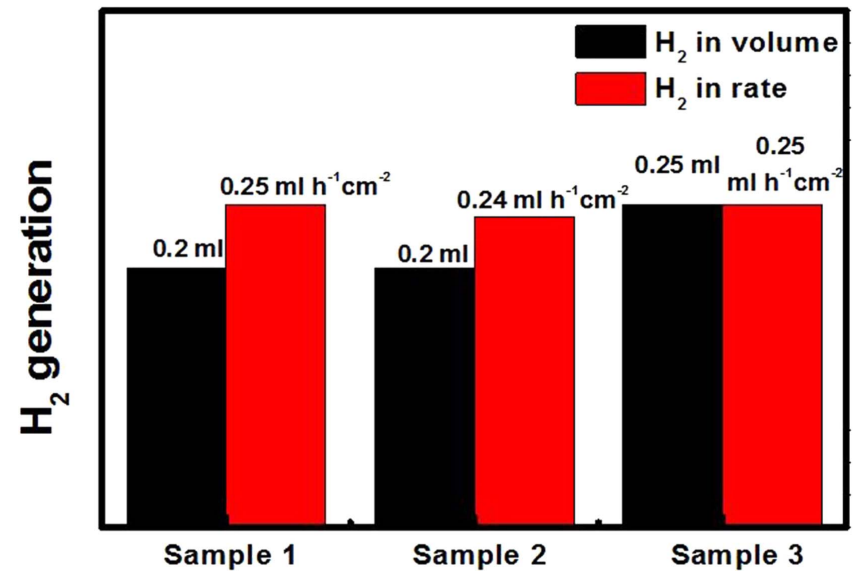

Figure 6. $\mathrm{H}_{2}$ generation from three samples under a $200 \mathrm{~mW} \mathrm{~cm}^{-2}$ illumination at $0.8 \mathrm{~V}$, where the measured photocurrent density is $0.73 \mathrm{~mA} \mathrm{~cm}^{-2}$ for sample 1 as an example (black columns: the total $\mathrm{H}_{2}$ volumes collected from each sample; red columns: $\mathrm{H}_{2}$ generation rates).

with more than $1 \mathrm{~A} \mathrm{~cm}^{-2}$, and then slightly reduces to $0.89 \mathrm{~A} \mathrm{~cm}^{-2}$ after $30 \mathrm{~min}$, representing a photocurrent degeneration rate of $3.7 \mu \mathrm{A} \mathrm{cm}^{-2} \mathrm{~min}^{-1}$. The reduction of the photocurrent is likely related to a slow photoelectrochemical corrosion occurring on the working electrode, although it is known that defect-free $\mathrm{GaN}$ is chemically inert to strong acids [19]. During the photoelectrolysis of $\mathrm{HCl}$, chlorine produced at the working electrode will adhere to the surface of GaN nano-pyramids and react with $\mathrm{Ga}$ atoms through defects $[8,20]$. The resultant of halides $\mathrm{GaCl}_{x}$ could be dissolved in the acidic solution, generating $\mathrm{Ga}$ vacancies acting as recombination centres. As a consequence, the associated photocurrent will decrease. It is worth highlighting that further optimisation of the growth conditions of the $\mathrm{GaN}$ nano-pyramids would lead to improvement in crystal quality, thus efficiently reducing or eliminating such defect related photoelectrochemical corrosion.

In order to measure a $\mathrm{H}_{2}$ generation rate, we fabricated three samples with an optical active area of 0.8, 0.85 and $1 \mathrm{~cm}^{2}$ (labelled as sample 1, sample 2 and sample 3), respectively. All the samples were tested under an illumination intensity of $200 \mathrm{~mW} \mathrm{~cm}^{-2}$ at $0.8 \mathrm{~V}$ for $1 \mathrm{~h}$ in $1 \mathrm{M} \mathrm{HCl}$. The black columns in figure 6 indicate total $\mathrm{H}_{2}$ volumes collected from a Pt counter electrode for each sample; whilst the red columns are the corresponding $\mathrm{H}_{2}$ generation rates by considering the optical active areas. It is observed that all of the samples exhibit almost the same $\mathrm{H}_{2}$ generation rate of $\sim 0.25 \mathrm{ml} \mathrm{h}^{-1} \mathrm{~cm}^{-2}$, corresponding to a Faradaic efficiency of $0.75-0.8$. The rate is much faster than that of any planar GaNon-Sapphire devices (typically $\sim 0.1 \mathrm{ml} \mathrm{h}^{-1} \mathrm{~cm}^{-2}$ ) [8], due to the aforementioned unique optical and electrical properties of our nano-pyramids.

\section{Conclusion}

In summary, we have reported a prototype photoelectrode fabricated from a GaN nano-pyramid array structure grown on cost-effective silicon substrates. The nano-pyramid configuration leads to significant enhancement in not only optical absorption, but also the transportation of photogenerated hole to semiconductor/electrolyte interface. A high hole transfer efficiency has been observed in our photoelectrode, which enhances the photocurrent. Consequently, the photoelectrode demonstrates a high photocurrent density of $1 \mathrm{~mA} \mathrm{~cm}^{-2}$ under a $200 \mathrm{~mW} \mathrm{~cm}^{-2}$ illumination and a large IPCE of $46.8 \%$ at $365 \mathrm{~nm}$, the bandgap of $\mathrm{GaN}$. We believe this 
structure opens up a way for the fabrication of $\mathrm{GaN}$ based photoelectrodes with a high energy conversion efficiency for solar-powered water splitting.

\section{Acknowledgments}

This work was supported by the UK Engineering and Physical Sciences Research Council (EPSRC).

\section{References}

[1] Juodkazytė J, Šebeka B, Savickaja I, Kadys A, Jelmakas E, Grinys T, Juodkazis S, Juodkazis K and Malinauskas T 2014 Sol. Energy Mater. Sol. Cells 13036

[2] Liu S Y, Sheu J K, Lin Y C, Chen Y T, Tu S J, Lee M L and Lai W C 2013 Opt. Express 21 A991

[3] Moses P G and Van De Walle C G 2010 Appl. Phys. Lett. 96 021908

[4] Maeda K, Teramura K, Lu D, Takata T, Saito N, Inoue Y and Domen K 2006 Nature 440295

[5] Waki I, Cohen D, Lal R, Mishra U, Denbaars S P and Nakamura S 2007 Appl. Phys. Lett. 91093519

[6] Benton J, Bai J and Wang T 2014 Appl. Phys. Lett. 105223902

[7] Benton J, Bai J and Wang T 2013 Appl. Phys. Lett. 103133904
[8] Benton J, Bai J and Wang T 2013 Appl. Phys. Lett. 102 173905

[9] Wang D, Pierre A, Kibria M G, Cui K, Han X, Bevan K H, Guo H, Paradis S, Hakima A R and Mi Z 2011 Nano Lett. 112353

[10] Jeong S, McGehee M D and Cui Y 2013 Nat. Commun. 42950

[11] Hou Y, Syed Z A, Smith R, Athanasiou M, Gong Y, Yu X, Bai J and Wang T 2016 J. Phys. D: Appl. Phys. 49265601

[12] AlOtaibi B, Harati M, Fan S, Zhao S, Nguyen H P T, Kibria M G and Mi Z 2013 Nanotechnology 24175401

[13] Fan S, AlOtaibi B, Woo S Y, Wang Y, Botton G A and Mi Z 2015 Nano Lett. 152721

[14] AlOtaibi B, Fan S, Vanka S, Kibria M G and Mi Z 2015 Nano Lett. 156281

[15] Lee S H, Zhang X G, Parish C M, Lee H N, Smith D B, He Y and Xu J 2011 Adv. Mater. 234381

[16] Shen S, Zhou J, Dong C L, Hu Y, Tseng E N, Guo P, Guo L and Mao S S 2014 Sci. Rep. 46627

[17] Dunn H K, Feckl J M, Müller A, Fattakhova-Rohlfing D, Morehead S G, Roos J, Peter L M, Scheu C and Bein T 2014 Phys. Chem. Chem. Phys. 1624610

[18] Chen R S, Chen H Y, Lu C Y, Chen K H, Chen C P, Chen L C and Yang Y J 2007 Appl. Phys. Lett. 91223106

[19] AlOtaibi B, Nguyen H P T, Zhao S, Kibria M G, Fan S and Mi Z 2013 Nano Lett. 134356

[20] Iniewski K 2011 Nano-Semiconductors: Devices and Technology (Boca Raton, FL; London: CRC Press; Taylor and Francis) p 430 